\title{
DEUX CENTRES CATALANS \\ AU MOYEN ÂGE: \\ BARCELONE ET PERPIGNAN
}

Philippe Wolff

Le caractère catalan de Perpignan est hors de discussion. L'Histoire de Perpignan, que j'ai l'honneur de diriger, moi venu des «brumes du Nord», mais si attaché à la Catalogne et à ses historiens, est parue le 23 avril 1985, au jour de la Sant Jordi, qui est, à Perpignan comme dans le reste de la Catalogne, grande fête pour les gens du livre'. Certes, depuis 1659, le Roussillon est rattaché au royaume de France; il ne s'y est pas résigné sans regrets ni sans révoltes. Et puis, la francisation, soutenue par le centralisme monarchique, puis jacobin, a progressé lentement, mais sûrement. La ville de Perpignan y est même venue plus tôt que le reste du Roussillon. De nos jours, un réveil catalan s'y est, en revanche, manifesté. Si ses implications politiques peuvent faire l'objet de controverses, par contre son aspect culturel n'est pas niable. Depuis 1981, une Université de Perpignan

1 Toulouse, editions Privat, 310 pages, illustré. Le chapitre sur Ruscino y est l'oeuvre de M. Rémy Marichal, auquel les lignes qui suivent doivent beaucoup. M. Pierre Ponsich et moi même nous y sommes partagé l'histoire médiévale, Marcel Durliat y a traité de l'art. La bibliographie n'est évidemment pas aussi simple pour Barcelone. Même en s'en tenant à l'essentiel, que d'ouvrages seraient à citer, d'Antoni de Capmany à Francesc Carreras Candi, de Jaume Vicens i Vives a Claude Carrère, entre autres... 
est même officiellement reconnue. Voilà qui nous reporte au Moyen Âge, alors que Perpignan, bien avant Barcelone, possédait une Université. Il est donc naturel que, pour honorer le collègue et ami que, depuis de nombreuses années déjà, fut pour moi le Dr. Frederic Udina Martorell, j'aie songé à lui brindar cette comparaison entre deux villes que tant d'aspects assimilaient et opposaient à la fois, au Moyen Âge. Aucune mort ne s'ensuivra, on peut du moins l'espérer. Mais cette modeste contribution aura montré l'affection et l'estime que, comme il va de soi, je porte à notre directeur honoraire de l'Arxiu de la Corona d'Aragó, où il m'a si souvent reçu avec son habituelle courtoisie, et où nous avons travaillé ensemble.

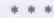

Ce sont d'abord leurs origines qui contrastent fortement entre les deux cités - non sans cependant que quelques caractères communs puissent s'y déceler. On m'excusera de les rappeler très brièvement pour Barcelone.

Sur le site même qu'occupe aujourd'hui le «barri gòtic», et que désigne encore au touriste une légère éminence, Barcino a été capitale d'un peuple indigène, les Laetani. Elle a subi des influences grecques et phéniciennes, qu'il n'est pas question de traiter ici en détail. Notons seulement qu'elle n'a pas été un centre de la colonisation phocéenne, relayée par Marsella (Marseille), et illustrée surtout par Roses et Empúries. Plus tard, les Grecs on été continués par les Phéniciens, qui avaient trouvé dans Carthage un reflet vivant bientôt de sa vie propre, et créant la colonie de Carthagène. Et c'est enfin par la rivalité entre Carthaginois et Romains que Barcino est entrée dans l'histoire du monde: ils s'étaient mis d'accord pour limiter leurs zones d'influence à la cité de Carthagène (348 av. JC), puis à l'embouchure de l'Ebre (226 av. JC). On sait qu'Hannibal viola cet accord en franchissant l'Ebre (218), et que les Romains, débutant d'abord à Empúries, l'emportèrent finalement dans cette lutte à mort. Barcino reçut fortement l'empreinte romaine, dont les fouilles révèlent tous les jours les restes, tandis que l'on peut admirer 
une partie des murailles tardives et restaurées. Mais c'est Tàrraco qui fut d'abord la capitale des Romains. C'est ici que se dressent les plus beaux de leurs monuments. Le christianisme y pénètre; saint Paul peut-être, sant Cugat et sant Feliu certainement, l'ont prêché, à Barcino sans doute comme à Tàrraco. Les sants Fructuós, Auguri et Eulogi ont subi le martyre dans celle-ci, et santa Eulàlia dans celle-là (vers 250). Toutes deux deviennent alors des sièges d'évêchés. Le regretté Ferran Soldevila soulignait les avantages et les inconvénients que comporta pour les Catalans cette influence romaine:

Va afavorir, hem dit, el procés d'unificació de les terres catalanes: apareix, per primera vegada, una entitat que, en una forma o altra, relliga i plasma Catalunya, si bé encara no amb el seu nom. Aquesta entitat és de caràcter judicial: el convent juridic tarraconense. Els seus límits no comprenen, però, totes les terres catalanes. És Catalunya; però una Catalunya, podríem dir, retallada. No en forma part el Rosselló, que resta inclòs dins la Gàl-lia Narbonesa; tampoc no en formen part les terres ponentines, que són adjuntades al convent jurídic cesaraugustà. Dos fets de remarcable importància, que subratllen fets geogràfics i, en algun aspecte, fets ètnics. Més encara: la part meridional del País Valencià - del Xúquer en avall- n'és també exclosa. I així mateix les Balears... Si Roma, amb el convent jurídic tarraconense, dóna, per primera vegada, forma a Catalunya, li prepara, també, dificultats per a la seva futura integració total. Són precedents polítics que poden influir en les ambicions polítiques dels pobles veïns. França, la monarquia francesa, tindrà com un dels seus grans ideals l'assoliment de les fronteres de la Gàl-lia romana; maldarà per aconseguir-les, i, per aquesta banda, hi reeixirà... En l'ordre exclusivament espiritual, la més forta influència de Roma es manifesta en l'idioma i en el dret... L'Església, a més a més, fou en molts aspectes com l'hereva de l'esperit de Roma i la seva influència, decisiva a Catalunya, com a tot l'Occident d'Europa.

Per la força de totes aquestes causes plegades, malgrat l'enfonsament de l'Imperi, malgrat l'al-luvió dels nous pobles, malgrat el pas dels segles, perdurarà a Catalunya, almenys com un ideal a aconseguir, a l'igual que als paisos més llatinizats del món romà, «el sentit d'autoritat, l'esperit de mesura i ordre, l'art d'administrar, que havien estat les grans creacions de Roma». $I$ algunes de les qualitats que les havien fetes néixer pugnaran 
sempre per obrir-se camí entre la tofa de qualitats adverses que tendiran a ofegar-les ${ }^{2}$.

Ce sont les mêmes faits, la même atmosphère, qu'a vécus, non pas Perpignan, qui n'existait pas encore, mais Ruscino, et beaucoup plus modestement.

Au cours du Bronze final, vers $1250 / 1200$ à $725 / 700$ av. JC, l'homme occupe un promontoire, qui se détache de la ligne de bordure de la terrasse longeant la Têt du site actuel de Perpignan à la mer, et se défend naturellement par des pentes abruptes. Sa position est relativement centrale dans la plaine du Roussillon, mais surtout il est sur l'axe de passage qui relie la péninsule italique et l'Europe du Nord à la péninsule ibérique. Cette occupation perdurera: ce sera Ruscino. Le choix de son emplacement laisse supposer que Ruscino contrôlera le franchissement du fleuve. Depuis environ 600 av. JC, elle importe des produits étrusques (donc d'Italie), puis de tout le bassin méditerranéen; elle subit ainsi fortement les influences grecques et carthaginoises. Elle est mêlée aux guerres puniques: Lorsqu'il franchit la Têt, Hannibal la saisit tout d'abord. Mais il n'y aura pas conquête militaire violente par les Romains. La romanisation se fera sans résistance ni brutalités.

Une vraie ville romaine se construit sur l'emplacement actuel de Château-Roussillon. Depuis environ 20 av. JC, lui est donné un forum de plan classique, avec basilique civile; des maisons s'ouvrent sur une rue orientée est-ouest, qui correspond sans doute au decamanus maximus, recoupé par un cardo. Autour du changement d'ère, les habitations semblent se multiplier. Il s'agit de petites exploitations agricoles - comme le montre entre autres la présence de silos- que développe la mise en valeur de la plaine cadastrée. Une ville basse se construit même peut-être entre la colline et la fleuve. L'apogée de Ruscino coïncide avec le gouvernement des empereurs julio-claudiens. Aussi subitelle les effets de la crise de 68 ap. JC, qui voit la chute de Néron et la prise de pouvoir de Galba. Elle devient bourgade sans importance,

${ }^{2}$ Història de Catalunya, Alpha, Barcelona 1962, 2a. edició, pp. 16-20. 
sous le contrôle de Narbonne. Pour autant que permettent de le constater les fouilles menées dans un site ultérieurement perturbé, tout peuplement en disparaît même vers la fin du ler siècle. Rossillo devient une simple forteresse, détruite sans doute par un raid normand en 589.

La villa Perpiniani apparaîtra en 927 dans un texte comme domaine rural tant les comtes de Roussillon sont seigneurs. Ils y fixent leur résidence à la fin du 10e siècle. Au début du 1le, une ville nouvelle s'y développe. Ả quand remonte l'occupation, de ce nouveau site? Comment s'explique le transfert, par des raisons géographiques (un choix plus judicieux de l'emplacement) ou religieuses (le passage d'un chemin de pèlerinage comme à Montpellier?)? Il y a ici, en tout cas, un phénomène assez rare dans l'histoire française, et qui distingue nettement Barcelone, où tout s'est accompli sur place, de Perpignan, ville à la fois très ancienne - si l'on accepte la tradition de Ruscino- et relativement récente dans son actuel emplacement.

Lévolution comparée des deux villes, sur les plans politique et religieux, offre aussi nombre de contrastes dans un parallélisme qui n'en est pas moins certain - croissance et relative libération étant le fait des deux, mais de façons différentes.

Au sein du monde romain, Barcelone n'a pas été tôt ruinée comme Ruscino. Elle s'y est au contraire développée. Aux 3e et $4 \mathrm{e}$ siècles, une fortification d'une remarquable puissance lui a été donnée, dont les murs et les tours ont été en partie construits à l'aide de matériaux arrachés aux monuments détruits de la cité romaine. Sous les Wisigoths a perduré la ville forte des Romains: en 415 y est mort le roi Athaulf, venant de Rome prise et pillée. Puis ce peuple s'est fixé peu à peu, prenant bientôt Tolède comme capitale. C'est la conquête «arabe» qui assura la primauté de Barcelone sur Tarragone. Avant la fin de 714, la zone «catalane» fut parcourue dans son ensemble par Arabes et Berbères, qui conclurent des traités avec les villes, donc sans doute aussi avec Barcelone. On sait qu'ils s'aventurèrent jusque dans la future France, et qu'en 732 une de leurs troupes - sans doute une for- 
te reconnaissance - fut mise en déroute près de Poitiers par Charles Martel, ancêtre des Carolingiens. Les Francs passèrent assez naturellement à la contre-offensive. A l'issue de plusieurs campagnes dévastatrices, Pépin le Bref, fils de Charles Martel, se rendit maître de la Septimanie, partie du royaume wisigothique située au nord des Pyrénées et Albères, occupée par les Musulmans (actuel Languedoc oriental - 752-759). Plus tard Charlemagne, fils de Pépin le Bref devenu roi des Francs, reçut à Paderborn, dans cette Saxe qu'il était en train de conquérir péniblement, à coups d'efforts et de sang (le sang des autres, en grande partie) plusieurs chefs musulmans révoltés contre l'émir de Cordoue, dont le wali de Barcelone, Sulaïman-ibn-al-Arabi, qui promit de lui livrer les villes en son pouvoir. Charlemagne voulut-il profiter des dissensions de l'Islam ibérique pour établir, entre Ebre et Pyrénées, un glacis protégeant son royaume? Nourrissait-il de plus amples desseins encore, l'établissement d'un protectorat sur Cordoue? L'importance des moyens qu'il mit en oeuvre permet de se poser ces questions. Mais cet homme du Nord était vraiment trop naïf, il n’avait pas compté avec la versatilité de ses pseudo-alliés islamiques, et il dut promptement sonner une retraite illustrée par l'épisode bien involontairement célèbre de Roncevaux (777-778).

Tout n'est cependant perdu. De nombreux Hispani, compromis dans l'affaire, se réfugient en Septimanie, où dès 782 ils sont assez nombreux pour obtenir un statut particulier. Ils se font les agents d'une active propagande en faveur de la libération de l'Espagne. Encouragées ainsi, les populations du Nord hispanique entreprennent de se libérer elles mêmes: les habitants de Gérone livrent leur ville à Charlemagne (785), imités bientôt par ceux de l'Urgell et de la Cerdagne (789). Cette sournoise avance de la domination franque provoque une réaction de l'émir Hishem Ier, dont en 793 une armée s'empare de Gérone et va ravager les faubourgs de Narbonne. Excellent prétexte pour le souverain franc. Le roi d'Aquitaine, son fils Louis (le futur Louis le Pieux) lance une offensive en direction de Barcelone, qui capitule en octobre 801; un accord assure aux indigènes un véritable statut juridique (exemption fiscale, obligations militaires, usage de leur 
propre loi). C'est en vain que Charlemagne cherche à aller plus loin. Pour trois siècles, la frontière de la Chrétienté se situera à quelques $\mathrm{km}$ au sud de Barcelone, aux abords du massif du Garraf et le long du Llobregat. Tarragone restera au Sud, obscur tas de pierres sous domination musulmane. Les cinq évêchés «libérés» (Barcelone, Gérone, Ausone, Urgell, Elne), autrefois suffragants de Tarragone, sont rattachés à la métropole de Narbonne. Barcelone devient «capitale» d'un comté, qui peu à peu unifiera la Catalogne.

Qu'on m'excuse d'avoir si longement insisté sur des événements, qui en fait jouent dans le devenir de Barcelone un rôle considérable. Car aucune comparaison n'est possible. Perpignan n'y a pas pris part, puisqu'il ${ }^{3}$ n'existe pas, nous trouvons justement dans la période de vide qui sépare Ruscino de Perpignan. Le Roussillon, lui, existe, mais son évêque réside à Elne. Son comte, lui, erre, ne disposant pas comme son cousin de Barcelone d'un grand centre urbain prêt à l'accueillir; ce n'est que vers la fin du 10e siècle qu'il se fixe à Perpignan.

C'est précisément alors que se situe un autre événement décisif pour l'histoire de Barcelone: en 985, Al-Mansour, maître de Cordoue, prend d'assaut et incendie la cité catalane, tue ses habitants ou les mène vers le Sud en un pitayable cortège. C'est en vain que Borrell comte de Barcelone fait appel au roi franc dont théoriquement il dépend toujours; Hugues Capet se borne à promettre son aide assortie d'une demande d'hommage. Rien n'en sort, et dès lors les Catalans se considéreront comme hors du royaume de France. Perpignan n'a évidemment pas connu les mêmes alarmes; au mieux dirons-nous plaisament qu'il était alors dans le sein de sa mère.

$$
\text { *** }
$$

Mais bientot naît Perpignan, et les évolutions politique et religieuse des deux villes peuvent se comparer. En 1010, l'expédi-

${ }^{3}$ En français, les noms de ville sont du féminin quand leur dernière syllabe contient la lettre e; sinon, il faut employer le masculin. 
tion contre Cordoue, qui réunit les comtes de Barcelone, Urgell et Besalú, les évêques de Barcelone, Elne, Gérone et Vic, est bien «le premier acte d'affirmation collective du peuple catalan» (Ramon d'Abadal) ${ }^{4}$. Cependant, la maison de Barcelone ne connaît encore qu'une prééminence familiale, qui va lui permettre de regrouper peu à peu l'ensemble de la Catalogne. Particulièrement fructueux sera le long règne de Ramon Berenguer III (1097-1131). C'est alors cependant que l'offensive almoravide menace les comtés catalans; Barcelone est même assiégée en 1115. En revanche, le comte barcelonais prend la tête d'une flotte internationale réunie pour réduire la piraterie des Baléares. Palma est prise en avril 1115; cette conquête d'un jour annonce les destinées maritimes de Barcelone. Et ce règne permet à l'unification catalane d'accomplir des progrès importants: en 1111, résultat du stérile mariage qui a uni le dernier comte de Besalú et une fille de Ramon Berenguer, ce comté est intégré à la maison de Barcelone; elle hérite en 1117 du comté de Cerdagne, dont le dernier titulaire meurt sans héritier.

Capitale du Comté principal de Catalogne, Barcelone profite de sa progressive ascension. En 1137 -date capitale- Ramon Berenguer IV se voit promettre la main de Pétronille, la très jeune héritière de l'Aragon. Si jeune que le mariage ne se consommera qu'en 1150. Mais Ramon Berenguer IV inaugure la dilatation de l'Etat catalan, auquel il su, au sein de la Couronne d'Aragon, conserver son identité propre. Il s'empare de Tortosa et de Lleida, puis de la «Nouvelle Catalogne». Il reconstitue la province ecclésiastique de Tarragone, dont seule Elne ne relève pas. Il dote son Etat catalan des Usatges de Barcelona. La voie des grands comtes-rois est ouverte.

Les destinées politiques de Perpignan sont moins brillantes.

${ }^{4}$ Sans vouloir donner une véritable bibliographie, je ne puis laisser passer ce nom sans dire ce que l'exposé de quelque sujet catalan que ce soit, depuis les Wisigoths jusqu'au $14 \mathrm{e}$ siècle inclusivement, doit à l'oeuvre de ce grand historien. Je me borne à renvoyer aux bibliographies spécialisées. Je voudrais mentionner également un autre nom qui domine l'histoire médiévale de la Catalogne, PIErre BonNassie, La Catalogne du milieu du Xe à la fin du XIe siècle, Croissance et mutations d'une société, Toulouse 1975-76, 2 vol. 
Certes il devient vraiment la «capitale» (dans le sens encore modeste de ce mot) du comté de Roussillon, il devient rapidement une véritable ville. En 1172, le comte Girard II accomplit le pas décisif: il lègue le Roussillon à Alfonse II (Ier en Aragon), comte de Barcelone et roi d'Aragon, qui prend bientôt possession de la ville. Dès lors, Perpignan suit les destinées de l'Etat catalan. Alfonse II -N'Anfos, comme on l'y appelait familièrement $-s^{\prime} y$ rend très populaire, $y$ séjourne fréquemment, $y$ meurt en 1196. Son fils Pierre II (Ier) s'efforce d'y continuer sa politique. Si sa mort tragique et prématurée à Muret (1213) ouvre une crise, durant laquelle Nunyo Sanç, comte de Cerdagne et Conflent, agit aussi en comte de Roussillon, tout rentre dans l'ordre à sa mort, en 1241. Jacques le Conquérant vient alors (1243) prendre possession du comté et de la ville. C'est lui cependant qui, peu avant sa mort, assume la décision si grave pour les deux villes, partageant ses Etats entre ses deux fils Pierre et Jacques: au premier la Couronne d'Aragon proprement dite, au second un royaume de Majorque, composé des Baléares, du Roussillon, de la Cerdagne et de la vicomté de Carlat. Perpignan en devient alors la capitale, et connaît son apogée médiéval - artistique et spirituel aussi bien que politique. Éclat éphémère. Dès 1295, Jacques II de Majorque prend ouvertement le parti du roi de France dans le conflit qui oppose celui-ci à Pierre II (III) le Grand. La défaite du premier sonne le glas de cette fragile construction qu'était le royaume de Majorque, et qui s'achève par la cession de Montpellier au roi de France (1349). Dès 1344, Pierre III (IV) le Cérémonieux a pris - non sans peine- possession du Roussillon et de Perpignan. Celui-ci va des lors partager le destin de l'Etat catalan, et y retrouver, loin derrière Barcelone, sa place naturelle.

De cette évolution politique, je voudrais maintenant détacher ce qui concerne l'administration des deux villes. Ici, Perpignan fait preuve d'une étonnante antériorité par rapport à Barcelone. C'est en effet dès 1197 que Perpignan obtient un consulat. Pierre II (Ier) l'octroya le 22 février de cette année: il y aura cinq consuls annuels, réeligibles, exerçant leurs fonctions à partir du ler mars. Tous les habitants se promettent, «tant les petits que 
les grands», de s'aider à défendre la ville, sauf contre le roi et les siens. Perpignan devient ainsi la première ville de consulat dans les pays catalans. On se perd en conjectures sur les motifs de cette décision de Pierre II, car les habitants n'ont en rien sollicité cette faveur. Il paraît probable que le but recherché était de mieux s'assurer la fidélité de la capitale du Roussillon, de rattachement récent, située aux confins d'un Languedoc plutôt agité. Ce consulat, les Perpignanais «semblent l'avoir reçu avec une certaine froideur et... ils l'administrèrent sans grand enthousiasme, du moins dans les premiers temps, car pendant plus de 50 ans aucun autre document n'y fait la moindre allusion" (Alart).

Tout autre est l'évolution à Barcelones. Dès le 1le siècle s'y sont formés les deux éléments dont Josep Maria Font i Rius a souligné l'importance dans l'élaboration du régime municipal catalan: des prud'hommes (probi ou boni homines) plus ou moins représentatifs, appelés à exercer les responsabilités en liaison avec les agents du comte; une communauté urbaine capable d'agir solidairement. Cependant c'est seulement le 17 avril 1249 que Jacques le Conquérant désigne pour un an quatre paciarii chargés de régler les affaires de la cité, avec l'aide de conseillers qu'ils pourront choisir. Un second acte, du 27 juillet 1249, modifie légèrement ce système à peine rodé, tout en lui donnant la durée. Il organise une commission exécutive de quatre paciarii désignée pour un an par les sortants; un Conseil assesseur composé de huit membres nommés par les «pahers»; une assemblée générale des habitants. Au prix de retouches opérées en 1257, puis en 1260,1265 et 1274 , ce système aboutit au régime durable des cinq conseillers élus à deux degrés par le Conseil des Cent.

Pourquoi ce retard de près d'un demi siècle sur Perpignan? Evidemment pas par un moindre progrès de l'économie et de la société barcelonaises. La pression de l'opinion publique? Nous

5 Sur cette question, je me permets de renvoyer à ma modeste contribution au 7e Congrès d'Histoire de la Couronne d'Aragon, «Barcelone et Toulouse aux XIle et XIIle siècles» (VII Congrés d'Història de la Corona d'Aragó, Crónica, Ponencias y comunicaciones, vol. II, Barcelona 1962, pp. 587-592). J'y signale la bibliographie antérieure, fournie du côté catalan, en particulier les travaux de mon collègue et ami Josep Maria FONT I RIUS. 
avons vu ce qu'il fallait en penser pour Perpignan. Il devait en aller à peu près de même pour Barcelone. Nous autres historiens nourris du récit des luttes des $18 \mathrm{e}$ et $19 \mathrm{e}$ siècles avons tendance à considérer que les concessions de consulats étaient des avantages âprement recherchés; en fait, ils comportaient aussi des charges, auxquelles les habitants étaient au moins aussi sensibles. Alors, la grande raison nous apparaît aussi clairement: Perpignan était l'avant-poste du Nord, qu'il fallait se concilier par diverses faveurs; Barcelone la capitale, avec laquelle il convenait de se montrer bien plus circonspect.

Sur l'évolution ecclésiastique comparée, je pourrai me montrer plus bref. Barcelone est un important évêché, le clergé y est nombreux, la vie religieuse ardente et variẻe: n'ìnsistons pas, un volume entier n'y suffirait pas. Perpignan n'est pas un évêché, il ne le deviendra qu'en 1602, lorsqu'Elne devra renoncer à son siège multiséculaire. Mais cette absence d'un évêque est aussi un avantage, même du point de vue religieux. Plus grande y est la liberté. Dès 1116 y a été créé un Hôpital des Pauvres. A la paroisse primitive de Saint-Jean-Le-Vieux en ont été ajoutées d'autres, Saint-Jaques (mi-13e siècle), Sainte-Marie-la-Real et SaintMatthieu (1300). En 1324 est posée la première pierre de la nouvelle église de Saint-Jean-Le-Vieux, qui, bien plus tard, deviendra cathédrale. Surtout Perpignan est très mêlé au conflit qui, au sein de l'Ordre des Frères Mineurs, oppose partisans d'une conception étroite - les Spirituels - ou large - les Conventuelsde la règle léguée par saint François d'Assise. Que Perpignan se soit rangé du côté des Spirituels, des faits nombreaux l'attestent: le fils aîné du roi Sanç, Jacques, a préféré, à la succesion paternelle, la bure et les privations du couvent des Frères Mineurs de Perpignan. Son cadet Philippe, prêtre, membre du Tiers Ordre de saint François, n'accepte que par devoir de succéder à son père (1325); et, dès 1329 , il ira vivre en anachorète, en Italie. En 1331, le couvent perpignanais est le siège d'un chapitre général de l'Ordre, qui se prononce nettement en faveur des Spirituels.

Viennent les temps du Grand Schisme d'Occident. Barcelone et Perpignan seront du même côté. 
Il me reste à présenter rapidement ma comparaison sur les plans économique et culturel.

\section{$* * *$}

Sur le plan économique, le parallélisme est frappant. On me dispensera, je l'espère, de traiter ici de l'évolution économique de Barcelone depuis le 12 e siècle. Je me bornerai à en évoquer les grandes lignes pour Perpignan, et la comparaison se fera à peu près d'elle seule.

L'économie médiévale perpignanaise a connu des débuts modestes. Elle a du d'abord - ce qui n'était point original-assurer la subsistance des Perpignanais. Elle était donc en grande partie agricole. Quelques lueurs émergent du silence des trop rares textes. En 1094 est signalé un poids de Perpignan, en 1125 une «mesure» de Perpignan. Vers 1180-1190 sont créées deux foires de quinze jours, au Carême et autour de la Saint Barthélemy (24 août). Ce sont surtout des rendez-vous de marchands. Les marchands perpignanais fréquentent bien les foires de Champagne qui, dans le cadre européen, sont le rendez-vous suprême; on les y trouve mentionnés en 1261; ils s'y procurent surtout des draps flamands, les meilleurs de l'Occident. Mais le commerce perpignanais est principalement passif; on y attend les clients venus de l'extérieur, les marchands flamands justement, et ceux de Saint-Antonin en Rouergue, qui leur servent plus d'une fois d'intermédiaires. Il y a bien une modeste draperie locale. En 1249, Jacques le Conquérant en prend sous sa protection tous les ouvroirs (ateliers). Mais Perpignan reste surtout un centre commercial.

Tout va changer depuis 1285 , a la suite de la Croisade qui, non sans vicissitudes qu'il serait trop long de rappeler ici, coupe le Roussillon de la France. Dès lors, la draperie perpignanaise progresse rapidement. En 1308, dans l'acte d'achat de terrains destinés à l'installation de moulins drapiers, figurent 170 foulons. En 1317 le roi Sanç regroupe les tisserands de laine et de lin au Puig Sant Jaume, peu peuplé encore. Il était assez facile de se procurer la matière première: il y avait une laine locale abondante, la mise en culture du Roussillon associant une agricultu- 
re importante et un élevage prospère, qui fournit l'indispensable future à la premierére; on fait aussi appel à la laine du Maestrazgo, on acquiert des laines provenant d'Afrique du Nord, ou d'Espagne en général, où l'introduction du mouton mérino s'est montrée particulièrement bénéfique; on peut même acheter des laines anglaises, à Majorque ou en Angleterre. La demande accrue de plantes tinctoriales engage les propriétaires terriens de Perpignan à cultiver la roja (garance), le pastel - ainsi que les chardons propes au peignage.

Les documents notariés ne permettent guère de suivre les progrès de la qualité qui durent se produire. A Barcelone, la saie noire de Perpignan achetée en 1302 par le roi Jacques II et son frère Pierre pour porter le deuil de leur soeur Violante, première femme de Robert de Naples, fut payée 16 sous 6 deniers barcelonais la canne... Un drap bleu escarlat de Perpignan, vendu à raison de 16 sous la canne, servit à vêtir les trompettes du roi d'Aragon (M. Durliat). Concluens: une draperie de bonne qualité moyenne, comme à Barcelone.

Si la draperie perpignanaise, il y en a d'autres. Celle des cuirs. De façon plus générale, Perpignan est un très important centre de fabrication des basanes (alude) et d'autres pelleteries domestiques passées dans des bains d'alun; le faible nombre des artisans et la durée du travails exigé de chacun d'eux ont seuls limité cette production. On confectionne aussi des chapeaux de bièvre, souvent à partir des peaux de castor mais aussi de feutre, de lapin ou de chèvre.

Dès lors, il n'est plus guère question des foires de Perpignan, mais cette fulgurante ascension industrielle n'a en rien diminué le rôle commercial de la ville, bien au contraire. Elle en fait une place où les capitaux manquent, sont recherchés, et ceci y attire nombre d'investisseurs et de prêteurs.

De cet éclat - dont l'économie perpignanaise d'aujourd'hui, où Perpignan est un centre combien secondaire, ne pourrait certes fournir aucune idée- trois conséquences sont plus particulièrement à retenir:

1. Les essors économiques vont rarement sans accuser les inégalités sociales. Il en fut ainsi, en tout cas, à Perpignan comme 
à Barcelone, du $12 \mathrm{e}$ au $14 \mathrm{e}$ siècle. La même organisation de la société en «mains», la même évolution sociale du régime municipal sont à relever dans les deux villes ${ }^{6}$.

2. À côté des Chrétiens, les Juifs sont à la fois artisans et bénéficiaires de cet essor. L'historien américain Richard W. Emery a pu écrire: "la croissance de l'aljama à la fin du xime siècle n'est qu'un des aspects du développement de Perpignan». Ces Juifs sont une centaine de families vers 1300 , contre 140 à Narbonne: c'est ainsi l'une des communautés les plus nombreuses au nord des Pyrénées. Il s'agit d'un groupe très fluide, dont les membres repartent facilement vers leurs communautés d'origine. Ces Juifs vivent très peu du commerce extérieur, et jouent un rôle très faible dans le commerce local. Quelques artisans; quelques rabbis, lettrés et médecins. Mais ils sont avant tout des prêteurs, qui travaillent pour toutes les catégories de la population, à des villageois de toute la région, mais surtout aux importants marchands chrétiens. Aucun doute: sans les capitaux juifs, l'économie perpignanaise n'aurait pu fonctionner. Leurs fortunes, nous les connaissons surtout par les douaires qu'ils constituaient à leurs filles, très supérieurs à ceux des Chrétiens. En tout, vers 1300 , l'aljama devait être riche de 30.000 livres barcelonaises - soit en moyenne 300 par famille, sur la base de 100 familles. Comme à Barcelone, l'existence de cette prospère aljama provoqua bien des rancoeurs. La célèbre pogrom de 1391 fut loin d'atteindre à Perpignan la gravité qu'il connut à Barcelone. C'est que la bourgeoisie chrétienne était beaucoup plus capable de défendre ses prêteurs juifs. Le petit peuple antisémite y était aussi beaucoup moins fort et moins motivé. Aussi les conséquences furent-elles beaucoup moins redoutables; aucun mort, et en 1413-1414 l'aljama faisait encore relativement bonne figure: 150 familles possédant en tout une fortune d'environ 60.000 livres.

${ }^{6}$ Pour Barcelone, ce sont surtout les travaux de Carme BatLle GallarT (qu'elle poursuit avec son séminaire), qui dominent la question. Parmi ses ouvres essentielles, citons: Barcelona a mediados del siglo XV. Historia de una crisis urbana, El Albir, Barcelona 1976. Cet ouvrage publie tardivement le résultat de recherches poursuivies beaucoup plus tôt, comme le prouve entre autres: «La ideologia de la Busca", Estudios de Historia Moderna V, 1955. 
De toute façon, ce pogrom était - comme de nos jours nous n'en avons eu que trop d'épouvantables exemples - surtout le signe d'une crise sociale?.

3. Pas de vie économique sans monnaie. Cela est trop évident. Comme il est naturel, c'est en monnaie de la Couronne d'Aragon que se faisaient à Perpignan les transactions locales. La réforme prise ves 1260 par Jacques le Conquérant s'applique à Perpignan comme dans le reste de ses Etats: il établit alors la monnaie barcelonaise de tern, destinée à remplacer la monnaie doblen$c a$, dont les contrefaçons s'étaient par trop multipliées. Il est tout de même frappant que Pierre IV ait crée à Perpignan un atelier monétaire ( 7 août 1346), et l'ait chargé de frapper des florins d'Aragon, sur le modèle florentin, donc purs (à 24 carats), et pesant chacun un peu plus de 3 grammes 50 . On comprend cette décision: Perpignan, poste avancé vers le Nord, était bien placé pour recueillir le billon que l'on espérait faire venir de France. La décadence n'en est pas moins frappante. Dès 1352, en raison des diverses difficultés, la finesse tombe à 22 carats $3 / 4$. En 1362, en principe pour empêcher la fuite de l'or vers la France, la voici à 22 carats. En 1365 - suite de la guerre de Castille- elle passe à 18 carats. Puis la Monnaie de Perpignan cesse de frapper l'or.

Nous ne sommes pas beaucoup plus heureux avec l'argent. «Aux Corts de Barcelone en 1431-1434, les syndics de la cité se plaignent de ce que les monnaies d'argent battues par la secca de Perpignan ne sont pas du type réglementaire et n'ont pas la teneur légale de métal précieux; Pere Llobet, maître de la Monnaie de Perpignan, est sommé par le roi de paraître aux Corts et de s'en expliquer» (Claude Carrère) ${ }^{s}$.

7 Je me permets de renvoyer à mon article: «Le pogrom de 1391 en Espagne, Crise sociale ou non?», paru en anglais dans Past and Present, no. 50, février 1971, pp. 4-18; reparu en français dans Regards sur le Midi médiéval, Privat, Toulouse 1978, pp. 511-523.

${ }^{8}$ Le grand ouvrage de référence est ici celui de F. MATEu 1 Llopis, La moneda española, A. Martin, Barcelona 1946. Voir aussi; Earls J. HamiLton, Money, prices and wages in Valencia, Aragon and Navarre, 1351-1500, Cambridge (Mass.), 1936, XXVIII-310 pp. - sur lequel je me suis permis de faire des réserves: «Réflexions sur l'histoire des prix dans la péninsule ibérique aux 
Ainsi, secondaire par rapport à Barcelone, Perpignan lui est tout de même très semblable, et entretient d'ailleurs avec elle des relations suivies. Si elle bénéficie de certains avantages, elle le doit à cette position avancée vers le Nord. Nous l'avions déjà constaté sur le plan politique. Nous allons le constater à nouveau sur le plan culturel.

Il serait possible de parler très longuement de la vie culturelle de Barcelone, assez longuement encore de celle de Perpignan. Nous n'en ferons rien, et nous limiterons à ce qui concerne l'Université. Depuis 1300, la Catalogne ne possédait que le studium generale de Lleida. Rien de tel n'existait encore à Barcelone. Vu sa situation à la frontière du Languedoc, Perpignan parut bien placé pour drainer une partie de la clientèle des Universités de Montpellier et Toulouse et en détourner tous les Roussillonais. Aussi Pierre III (IV) y institua, par une charte du 20 mars 1350, un studium generale, c'est à dire où l'on pouvait étudier toutes les matières composant le curriculum universitaire. Parce qu'il était alors en mauvaises relations avec la Papauté, Clément VII lui fit attendre jusqu'au 28 novembre 1379 la bulle par laquelle il lui concédait le caractère universel indispensable à toute véritable Université, et ajouta l'enseignement de la médecine à celui du droit et des arts libéraux. La bulle supprimait, par contre, l'enseignement de la théologie, qui faisait partie de l'institution primitive: ce qui témoigne d'une méfiance dont il serait intéressant de connaître les motifs. Barcelone, faut-il le rappeler, attendra son Université jusqu'en 1533!

Il est grand temps de nous arréter. Nous pouvons dire en terminant que le sort ultérieur des deux villes est en germe au Moyen Age: Barcelone, capitale de la Catalogne, membre de la Couronne d'Aragon, persécutée par la Couronne de Castille - qui en 1659 abandonnera Perpignan et le Roussillon au roi de France.

XIVe et XVe siècles", Studi in memoria di Federigo Melis, Giannini ed., 1978, vol. III, pp. 73-90. 